\title{
Root Canal Treatment (RCT): From Traditional Endodontic Therapies to Innovating Pulp Regeneration
}

\author{
Michel Goldberg* \\ Professor Emeritus, Faculty of Fundamental and Biomedical Sciences, INSERM UMR
}

Received: June 10, 2016; Accepted: July 05, 2016; Published: July 13, 2016

*Corresponding author: Michel Goldberg, Professor Emeritus, Faculty of Fundamental and Biomedical Sciences, INSERM UMR -S 1124 and University Sorbonne City Paris , 45 rue des Saints Pères, 75270, Cedex 06, Paris, France; Tel- +336626767 09; Fax- +331495833 29, E-mail :michel.goldberg@ parisdescartes.fr, mgoldod@gmail.com

\begin{abstract}
Traditional protocols involve the cleaning of the pulp chamber and root canals, the removal of bacteria and of the smear layer, and ultimately to the sealing of endodontic materials filling the pulp, and root lumen. Disinfection combined with mechanical enlargement allows occluding the lumen of the dental pulp with a central master cone, and lateral condensation using a series of small accessory cones. Innovative approaches propose bioengineered scaffolds, growth and transcription factors. Removal of the smear layer reveals different types of globular and non-globular dentins (osteodentin, osteodentin, fibrodentin), cleaning the dentinal walls before chemical or mechanical enlargement of the lumen. Three distinct options open gates for classical endodontic therapies before conventional filling. New pulp therapies derived from apexogenesis and apexification contributes to the formation of a renewed dental pulp, involving totipotent, multipotent and unipotent stem cell. Proliferation of structural cells, signaling factors, transcription factors, metalloproteinases, and cytokines are involved in pulp renewal. Coronal and root microvascularization participate to pulp regeneration and ultimately to pulp mineralization. BMP-2, TGF- $\beta 1$, MTA and Portland cement may also contribute to the requirements for pulp bioengineering, leading to the future of innovating biological pulp therapies.
\end{abstract}

\section{Introduction}

For many years, Root Canal Treatments (RCT) used traditional protocols imposing the careful cleaning of the canals, the removal of the smear layer, and the sealing of the filling materials inside the pulp chamber $[1,2]$. These procedures were combined with the manual, instrumental or chemical shaping of the root lumen [3].

The preparation of the canal(s) includes chemical mechanical enlargements. We also took into account the coordination of the successive steps, leading ultimately to the preparation of the root canal before filling the empty space. After disinfection, pulp remnants were removed. A clean root canal was obtained after root widening. Endodontic cements filled the pulp chamber, namely when a zinc oxide eugenol mixture was used. Employing a central master cone, and a series of small accessory cones, after axial and lateral condensation the root chamber was gradually filled. These steps include the sealing of lateral, secondary and accessory canals [4].

In addition to conventional protocols, more recently attempts were made to regenerate the dental pulp. A few pulp cells proliferate, and renewed the pulp tissue. We envision the formation of a dental pulp and furthermore its mineralization occluding the root lumen. Part of the endodontic cavity was still alive, or proliferation of pulp cells was requested to restore the biology of the dental pulp. Extracellular matrix molecules and/or other growth or transcription factors contributed to regenerate the pulp. In contrast with mechanical methods, biological options were considerate. The innovative approaches proposed were envisaging the implication of structural cells and signaling factors. In this context, we used stem cells, bioengineered scaffolds, growth factors, transcription factors, and cytokines. Altogether, the molecules were implicated in the different procedures that pave the way as biological substitutes for root canal treatments [5].

In the first part of this review, we summarize the basic concepts and related methods devoted to the traditional Root Canal Treatment (RCT). In the second part, we focus on recent concepts aiming to regenerate the dental pulp, leading to pulp renewal that further underwent mineralization. Along these lines of evidences, biological concepts provide the basis of innovating pulp therapies.

\section{Why, how and when traditional root canal treatments (RCT) are needed}

Dental caries are mostly due to bacteria and subsequently to pulpinfection [3]. Associated to the dental plaque; microorganisms alter enamel surface, either beneath the proximal contacts areas, or in the face-to-face enamelling occlusal fissures. The initial carious lesions is formed at this precocious stage.

The carious lesion crosses the whole enamel thickness and spread laterally along the enlarged dentino-enamel junction. The lesion degrades firstly the mantle dentin. Caries penetrate the subjacent circumpulpal dentin through dentinal tubules 
displaying enlarged diameters. After the destruction of peritubular dentin, measurements of the tubule diameter increase from 2 to $6 \mathrm{~m}$. Bacterial contamination is initially limited to the coronal pulp. During the next step of the disease, microorganisms invade the pulp, spread along the tubules, and penetrate into the main root canal. Afterward, they reach the apical pulp [6].

The progresses of carious lesion leave pulp remnants, and altered pulp cells. Fragments of the disorganized extracellular matrix and pulp debris persist in the pulp chamber. These processes are associated with vascular thrombus, neuronal pain and gradual degradation of the dental pulp. This cascade of events leads to the therapy of the dentin lesion, associated with the biocompatibility or cytotoxicity of restorative biomaterials. Gradually, this underlines the potential for direct or indirect pulp capping, coronal pulpotomy and ultimately the requirement of endodontic treatments [3].

To summarize, traditional Root Canal Treatments (RCT) are mostly due to the development of carious lesion. Gradually bacteria invade the dental pulp. Partial or total destruction of the pulp pave the way for the total degradation of the pulp. Small abscesses are formed some distance away of the odontoblast/ Hoehl's cells layers that are present at the outer surface of the pulp. The destruction of the odontoblast and sub-odontoblastic layers is firstly limited, expands and moves toward the apical part of the root. Micro-abscesses are increasing in size. They contaminate and degrade the coronal part of the pulp. Later, they diffuse in the root. Bacterial penetration is initiated by the carious decay, and diffusion occurs through the dentinal tubules. Finally, the lesion spreads into the root, and contributes to the total destruction of the dental pulp. At that stage, disintegration of the dental pulp implicates steps that dictate future therapeutic orientations.

Three commitments apply to the different pulp pathologies:

1) Deep carious lesion influence directly (partially or totally) or indirectly pulp capping [4]. They contribute to the formation of reactionary or reparative dentinal bridge. The dentin produced is very similar to osteodentin.

2) Surgical pulpotomy allows the preservation of a pulp still alive in the root. This step is associated with chemical disinfection. However, the cleaning solution should not be too heavily cytotoxic, and allows the preservation of cell vitality.

3) The third and ultimate step consists in a total pulpectomy. This last possibility implicates either a conventional endodontic treatment, or pulp regeneration. An endodontic filling (gutta percha, endodontic paste containing zinc oxide, associated with axial and lateral condensation) theoretically supports this ultimate step. Pulp cells colonized the dental pulp and contribute to the formation of a dense extracellular matrix. Pulp regeneration follows pulp renewal, and subsequently root canal mineralization [5].

Only a few lesions have a traumatic non-infectious origin, whereas most pulp lesions result from carious infections.
Endodontic treatments implicate consecutive critical steps. Following a cascade of preventive actions, endodontic therapies are associated with acute pulpitis, and with the terminal degradation of the pulp tissue. Periapical lesions are rapidly diffusing, reaching chronicity at some later stage. These procedures restrain infections. They invade the complex root canal system. Such lesions limit the spread of the lesion to the surrounding tissues (granuloma, cysts, and osteolytic lesions). All the methods used during these procedures involve irrigation solutions (sodium hypochlorite, and/or calcium hydroxide), which constitute selected antimicrobial agents. They contribute to clean the pulp walls of the pulp chamber, both in the coronal and in the root parts of the teeth [6].

Using traditional root treatment, the various techniques so far used include hand files (mechanical methods), or $\mathrm{NaOCl}$ and $\mathrm{H}_{2} \mathrm{O}_{2}$ (chemical disinfection). These methods are using alternatively irrigation, and they aim at removing the smear layer [7]. They precede chemical and mechanical enlargement. These step-by-step methods are followed by axial and lateral condensation. Sealing the master cone following the Root Canal Treatment (RCT) allows the apical closure of the main root canal. Compared to a group using contemporary methods (hand/rotary and NiTi files, NaOCl, bacteria-neutralizing chlorhexidin, EDTA and $\mathrm{H}_{2} \mathrm{O}_{2}$ irrigation), endodontic therapies favor warm and/or lateral condensation. A second group of experimental endodontic therapies has the benefit of a surgical microscope, and/or ultrasonic instrumentation, electronic apex location and digital radiography. Despite technological specificities, the two groups of treatments did not evidence statistically significant differences between the two different groups $[4,8]$.

\section{Removal of the smear layer}

It is mandatory to suppress the smear layer during the initial cleaning associated to endodontics treatment. The smear layer contains bacteria and by-products, preventing the penetration of filling materials into dentinal tubules, hence leaving an unwanted gap between the filling of the root and dentin walls. The smear layer contains organic remnants of odontoblastic processes, pulp tissue and bacteria. Inorganic in composition, this layer is present in dentinal tubules up to a depth of $40 \mathrm{~m}$ [9].

Morphological data implicate the opening of dentinal tubules, and the presence in the labial and lingual segments of root surfaces of globular and interglobular dentin. Globular structures provide evidence for osteodentin. By contrast, the mesial and distal surfaces look flat, amorphous, and deprived of tubules. In consequence, these surfaces display afibrodentin appearance.

Smear layer removal is mandatory in order to clean the dentin surfaces of the root, and open dentin tubuli. The cleaning process associated with disinfection includes chemical, ultrasonic and laser techniques. Mechanical enlargement of the main canal diameter contributes to eliminate the smear layer, and provides a homogeneous view on the dentin walls, including in sights on dentinal tubules. 
Chemical removal is essential to remove pulp debris associated with the smear layer created by the instrumentation process. Sodium hypochlorite used during or after instrumentation produces clean dentin walls. Chelating agents based on EDTA decalcified dentin remove dentinal plugs and cell remnants up to a depth of 20-30 m for treatment lasting about 5 minutes. The addition of a quaternary ammonium bromide (cetrimide) increases the elimination of the smear layer, opening the lumens of dentinal tubules. They reveal a ring of peritubular dentin around the lumens of the tubules. Organic acids used at $10 \%, 25 \%$ and $50 \%$ citric acid concentration are efficient for the preparation and cleaning of dentin surfaces. Ultrasonic removal of smear layer combined with $\mathrm{NaOCl}$ may be activated by an ultrasonic delivery system. Laser removal vaporizes tissue in the main canal, disrupt the smear layer, and melt the superficial structure. Recrystallization process is associated with the Er:Yag laser. The main canal is easier to penetrate when the available probes removed the smear layer $[7,9,10]$.

Cleaning the root canal: The smear layer is removed during chemo-mechanical preparation combined with ultrasonic irrigation, mainly when sodium hypochlorite is used at $60^{\circ} \mathrm{C}$ together with a $3 \%$ sodium hypochlorite combined with $17 \%$ EDTA for 1 minute. Large amount of dentin debris mixed with vital and necrotic remnants, mingled with microorganisms and microbial toxins, constitute fractions of the smear layer. They penetrate into dentinal tubules and are associated with the bacteria located near the border of the tubules. The removal of smear layer is never achieved totally in the apical third with these techniques, and it should be remember that the other protocols so far used remove the smear layer in the coronal and middle third of the root canal [7].

\section{Traditional root canal treatment using Thermafil and cold lateral condensation filling techniques}

Root Canal Treatments (RCT) using either ThermaFil (TF) or Lateral Condensation (LC) were compared. No statistically significant difference was detectable between the two groups [10]. After selecting the Master Cone (MC), the apical cone was sealed, and compressed laterally with spreaders. The process was repeated until the total RCT was achieved. After conventional filling, most of the treated teeth failed because vertical fracture occurred. The fissures were detected within the first two years following endodontic treatment. Up to $30 \%$ and $11 \%$ fractures were reported in teeth restored with amalgam and/ or after light-cured composite resin. Vertical fissures appeared after condensation of calibrated gutta percha cones inside the enlarged main canal. Fractures occurred probably because the overall dentin thickness was reduced by the canal preparation, and consequently the root was more easily broken.

\section{Endodontic Failures}

In some cases, RCT leads to a failure after endodontic treatments. It results from the microorganism colonies persisting in the apical portion. A whole list of traumatic failures is available. In addition to intraluminal endodontic infection, broken instruments, perforations, overfilling or under filling, are the main reasons of endodontic failures, even in apparently well-treated teeth. Bacteria located in areas such as isthmuses, ramifications, deltas, irregularities, niches and dentinal tubules seem to be not affected by endodontic disinfection procedures [11].

Treatments of teeth affected by deep caries preserve in the apical part of the root remnants that are still alive after indirect capping. Direct pulp capping with Calcium Hydroxide $(\mathrm{CH})$, and/or Mineral Trioxide Aggregates (MTA) constitutes actual therapies. They contribute to the formation of dentinal bridges containing tunnel defects, fissures, and bone-like osteocyte lacunae. Among the different options for the available therapies, pulpotomy (partial ablation of the pulp in the coronal pulp chamber) constitute a valid option. However, we give privilege to a solution removing totally the dental pulp. Chemo-mechanical and careful cleaning of the cavity (also named pulpectomy) constitutes a useful method. This end-point is very close to noninfectious conditions, as mentioned below [8].

The risk factors associated with endodontic failures increase the chance of success of a RCT (Root Canal Treatment). It is based on the accuracy of the initial diagnosis, the excellence of disinfection, the value of the instrumentation and filling procedures, leading finally to rehabilitation management. The treatment requires inactivation of microorganisms. It is mandatory to disrupt the bacterial biofilm. Antimicrobial strategies, root canal shaping, and coronal and apical seal appears to be key-factors for a successful treatment. Finally the rehabilitation management appears to be essential for the success of endodontic therapy. Absence of pain, regression of the bone lesion, tight seal of the canal and coronal spaces, recoveries of the tooth function are parameters that should be re-evaluated over time [5].

The basic aims of endodontic therapy are in distinguishable in adults and children [6,12]. Removal of the infection, and reduction of chronic inflammation are identical and follows the same lines of evidence. Three techniques have been proposed and they are requiring specific therapies:

Vital formocresol pulpotomy: The pulp tissue of deciduous teeth is dipped in a $1: 5$ dilution of Buckley's formocresol (tricresol 35\%, formaldehyde 19\%, glycerol 16\%, water 31\%).

Devitalization pulpotomy: The paste includes paraformaldehyde $1.00 \mathrm{~g}$, carbowax 1500: $1.30 \mathrm{~g}$, lignocaine $0.06 \mathrm{~g}$, propylene glycol $0.5 \mathrm{ml}$, and carmine $10 \mathrm{mg}$.

Non-vital pulpotomy: Beechwood creosote solution is sealed into the cavity with a zinc oxide-eugenol dressing (0-Methoxy phenol (guaicol) 47\%, P-methoxy phenol 26\%, 2-methoxy, 4 methyl phenol (Cresol) 13\%, M-methoxy phenol 7\%, other 7\%).

These three distinct options open gates for conventional endodontic therapies. Pulp regeneration and mineralization allows new therapeutic approaches. 


\section{Failure and success of conventional Root-Cana Therapy- toward regeneration of the dental pulp}

Examination of 104 cases of failure, shows that 66 were associated with a poor apical seal. It is obvious that necrotic debris and microorganisms cannot be completely eliminated from the prepared root canal. Chemically active, adhesive, rootcanal sealers minimize coronal microleakage [13].

When immature teeth are affected by caries or trauma, the pulp reaction requires management depending on the presence of inflammation and pulp vitality. Necrotic pulp, and root-end closure are required if the apex is not fully formed. Disinfection using sodium hypochlorite and calcium hydroxide (CT therapy) contribute to apexogenesis and to apexification [14]. This is depending on the closure or opening of the apical ending Closure results from the root canal therapy, whereas an open apex is related to the root-end closure and/or pulp regeneration. Complete root development requires a viable pulp containing cells that potentially differentiate into dentin-producing odontoblasts.

Stem cell-based regeneration of root apex may be mediated by adult stem cells. Stem cells have the capacity of self-renewal and multiple-differentiation (nerve, muscle, blood, lungs, pancreatic and skin cells). They can be divided into four stem cell types, including totipotent cells, pluripotent, multipotent and unipotent progenitors. HSCs isolated from bone marrow differentiate into all blood cells of the myeloid and lymphoid cell lineages.

MSCs (e.g. lineages of mesenchymal origin), as they were defined by Friedenstein, et al. [16] and later by Caplan [17], include bone, cartilage, fat, connective tissue, and muscle and marrow stroma. MSCs have a high proliferative potential. They express Oct-4, Nanog, STRO-1, CD73, CD90, CD105, and CD146. They are negative for CD14, CD34, CD45 and human leukocyte antigen-DR. Stem Cells of the Dental Pulp (DPSCs), from human exfoliated deciduous teeth, from the apical papilla (SCAPs), stem cells of the periodontal ligament, and dental follicle precursor cells still need to be better explored in order to define their potential capability during tissue therapies. They may be used in the context of pulp regeneration.

In addition to the traditional root canal treatment, structural cells, signaling factors, and a series of stem cells are concerned. They are implicated in pulp regeneration. Bioengineered scaffolds, growth factors, transcription factors, and cytokines are involved in pulp renewal. They are implicated in the differen procedures that play an actual role as biological substitutes for conventional root canal treatments.

\section{Discussion}

\section{Pulp regeneration, Stem cells and Extracellular matrix proteins}

The treatment of infected necrotic canal implicates the removal of remnants of the contaminated pulp. This involves careful disinfection, eliminating bacterial colonies. The end point of endodontic therapies is reached by the filling of the pulp chamber by zinc oxide, after condensation with gutta percha. In adults, regenerative strategies are required for the replacement of cells during root canal therapies.

Stem cells may be isolated and cultured from bone marrowderived and adipose tissue-derived mesenchymal progenitors. ECM molecules stimulate the increase of numerous precursor cells, leading the terminal differentiation of pulpoblasts toward dental pulp cells bearing a terminal phenotype. ECM form scaffolds containing associated structural and functional protein simplicated in pulp regeneration. They present a complete engineered pulp environment containing growth factors, cytokines and metalloproteases. The biomimetic ECM scaffold may trigger vascularization within the dental pulp.

ECM dental pulp stem cells may be used for pulp tissue engineering. Using somatic MSCs, root canal may regenerate a functional pulp, displaying sensitivity and vitality. DPSCs have been identified as sources for pulp renewal. There is no need for growth factors and/or differentiation factors to form a vascularized pulp-like tissue. Angiogenesis is a crucial step, and ECM scaffolds enriched with VEGF contribute actively to pulp regeneration. Coronal and root vascularization provide the scaffold that is needed for pulp renewal. Stem cells found in the apical part of the root proliferate, slide beneath the odontoblast/ Hoehl's layers, and participate to the fisher-like network located along the root. In the crown part, arteriolar, pre-capillaries and capillaries contribute to the formation of terminal loops, $100-150 \mathrm{~m}$ in diameter irrigating consecutive area. When the Raschkow's sub-odontoblastic plexus of capillaries irrigate the coronal part of the tooth, stem cells contribute in this context to rebuild a pulp that is still alive [18].

\section{Dental pulp regeneration and Mineralization}

Regeneration of the dental pulp appears as resulting from pulp degradation. Pulp degeneration by bacteria results from microorganism's invasion. Deep carious lesion leads to degenerative processes of the coronal pulp. Pulp alteration leads to a gradual disorganization of the connective tissue. Metalloproteases, such as collagenases and other MMPs are implicated in the degradation of the collagen network. Proteoglycans are subjected to the action of stromelysin, elastase and other ECM components also identified within the pulp. In addition, small peptides are cleaved and released from the ECM tri-dimensional architecture. Dissociation of fragments from the entire molecules contributes to the structural scaffold of matrix components. In the course of pulp degeneration, precocious destruction occurs firstly in the coronal pulp and later in the root part near the apical ending. Abscesses of limited size increases in size, merge and finally form large degradation areas. The end point of such degradation consists in pulp destruction.

The dental pulp includes colonies of fibroblast/pulpoblast cells. Intercellular gap junctional complexes link fibroblasts, binding together pulpoblasts and forming a continuous network. These cells are implicated in collagen synthesis and secretion. Pulp cells strongly linked move from the central part of the pulp 
toward the periphery. Other groups of cells involve macrophages. Inflammatory cells include immune cells, T-cells, helper/inducer $\mathrm{T}$ Cells, cytotoxic/suppressor $\mathrm{T}$ cells, macrophages and class II antigen-expressing cells. Dendritic cells, B-lymphocytes and T-lymphocytes are also found among pulp cells. Mast cells, dendritic cells are implicated in phagocytosis. They produce cytotoxic T-cells. They are natural killer T, usually producing chemokines and inflammatory cytokines. This group of cells appears to be also implicated in pulp regeneration [19].

In addition, microvascularization is produced within the pulp. Microvasculature implies arterioles, pre-capillaries, capillaries and venules. Pericytes are located around capillaries. Limited areas about $100-150 \mathrm{~m}$ wide, form bypass and arteriovenous anastomoses, contributing to the pulp chamber vascularization. By contrast, in the root a fisher-net organization allows drainage of the blood in the apical direction. Lymph vascularization collect the lymph, drained toward the apex via lymph capillaries [24].

A central innervation penetrates the dental pulp by the apex. Laterally, venules and arterioles fan out in the crown and form the subodontoblastic Raschkow plexus. Axons penetrate in dentin through the predentin, crossing intercellular junctions between odontoblast cell bodies. Axons penetrate into the inner dentin border along $150 \mathrm{~m}$ from the mineralization front. They are horizontally oriented and connect a few tubules.

Pulp Regeneration: Two distinct groups of progenitor cells have been identified. The first group concerns apical cells. In the apical part of the root (molar), cells are immunolabeled by the Proliferative Cell Nuclear Antigen (PCNA). They multiply and slide in the root pulp beneath the odontoblast/sub-odontoblast layers. PCNA labeled cells are located mainly in the apical part [20]. Six et al., [21] have shown that after 2 weeks, labeled cells become undetectable in the root. By contrast, labeled cells were located in the crown, around the agarose beads used as carriers. Beads loaded with labeled cells accumulate around amelogeninloaded beads. Hence, progenitor cells appear firstly in the root. Then they slide along the pulp chamber beneath the odontoblast/ Hoehl's layers. They contribute at later stages to label the crown part of the pulp. Sliding of the cells from the apex to the coronal part, near the exposure area, constitute the pathway that contributes to pulp regeneration $[14,15]$.

\section{Pulp capping: Selecting bioactive Materials and Molecules}

Using scaffolds containing growth factors, dentin regeneration was induced. Dental and non-dental clinical problems were clarified by using these alternative approaches, leading to mineralization, e.g. bone and dentin-pulp regeneration.

Calcium hydroxide $(\mathrm{CH})$ displays an alkali pH. However, $\mathrm{CH}$ produces inflammation within the underlying pulp. Tunnel defects are due to multiple tissue inclusions in the dentin bridge. The dentin bridge becomes permeable and allow bacterial invasion of the pulp. mRNA expression of Bone Morphogenetic Protein -2 (BMP-2) is enhanced. Notch 1 is expressed in the subodontoblastic zone, beneath lesions treated with $\mathrm{CH}$. Notch 1 and 3 are detected in perivascular cells.

Mineral Trioxide Aggregate (MTA) is an alternative pulp capping material. No necrotic tissue is found after MTA capping after 1-3 months. MTA induced ERKs activity The ERK/ MAPK pathway seems to be involved in the proliferation and differentiation of dental pulp cell. It may be a valuable alternative to the use of $\mathrm{CH}$ [22].

Portland cement is non-toxic and biocompatible. It induces reparative dentin formation in short-term evaluation. Used for dental root perforations, damages filled with Portland cement were clinically silent. A 9-years and 11-years follow-up appear compatible with normality. It is a non-irritant material that does not affect the structural integrity of cells.

TGF-betas are cytokines, members of the TGF superfamily. They induce odontoblast differentiation and dentin bridge formation. It up-regulates type I collagen expression in odontoblasts, via c-jun, a nuclear proto-oncogene, and further regulates an activation factor protein-1 (AP-1). Collagen membrane containing TGF- $\beta 1$ promotes dentin/pulp healing. BMP-2, BMP-4, and BMP- 6 are identified in human primary culture of dental pulp cells. Osteodentin formation in injured pulp is enhanced when treated by BMP-7. Osteopontin is implicated in reparative dentin formation $[18,23]$.

A significant reduction in the expression levels of growth factors BMP-4, -5, IGF-1, and TGF $\beta 1$ was observed, attributed to the down-regulation of RUNX2 and ECM protein biglycan, and ultimately with a reduction in type I collagen [18]. Fibroblast growth factor-2 (FGF-2) is involved in neo-vessel formation.

Bone Sialo-Protein (BSP) activates initial nucleation necessary for the mineralization process. MEPE, amelogenin and enamel matrix proteins are also useful. Pulp revascularization was seen clinically. Examination at intervals ranging from 6 months to 3 years was used, including a thickening of the dentinal walls as radiographically assessed. Alternative methods include plasma derived fibrin clots as scaffold and other methods of regeneration. Apexification procedures allow to produce apical development, either using calcium dihydroxide or MTA, together with a new mixture of antibiotics. Insulin-like growth factor-I is also implicated in reparative dentin formation. Pulp revascularization treatment depends on:

1) Root canal disinfection (even after irrigation with sodium hypochlorite at $2.5 \%$ ),

2) The presence of a scaffold (blood clot) and 3) hermetic coronary filling.

The generation of a functional tissue requires three key elements: stem cells, growth factors and a scaffold. The combined mixture of three antibiotics: minocycline, ciprofloxacin displaying an acid $\mathrm{pH}$, and metronidazole, the only antibiotic that display a neutral $\mathrm{pH}$, are implicated in the new clinical protocol proposed for pulp revascularization. The mixture has no cytotoxicity and is needed by stem cells [25]. 


\section{Conclusion}

To conclude, root canal treatment displays a number of successes and a few failures. Alternatively, the removal of the smear layer, enlargement of the main canal, axial and lateral condensation allows appropriate sealing of the lumen of the canal. It seems however, that reduction in thickness of the dentin layer leads to tunnel defects and vertical fissures. Pulp may regenerate, pulp cells have the capacity to proliferate, restore a pulp tissue, which will further mineralize. Pulp regeneration fulfills the requirements for pulp bioengineering. It opens gates for of pulp conventional treatments or leading for the future of innovating biological pulp therapies.

\section{References}

1. Goldberg M (a). Dentin, pulp, and tooth pain. In "Fundamentals of oral histology and physiology". First edition. AR Hand and Marion E Frank. Chapter 5. John Wiley \& sons, Blackwell inc. 2014; 85-112.

2. Goldberg M (b). Pulp anatomy and characterization of pulp cells. in: "Biology, pathology, and regenerative therapies". Springer Verlag, Berlin, Heidelberg 2014. 13-33.

3. Bergenholtz G, Spângberg L. Controversies in endodontics. Crit Rev Oral Biol Med. 2004;15(2):99-114.

4. Fleming $\mathrm{CH}$, Litaker MS, Alley LW, Eleazer PD. Comparison of classic endodontic techniques versus contemporary techniques on endodontic treatment success. J of Endo. 2010;36(3):414-418.

5. Bergenholtz G, Axelsson S, Davidson T, Frisk F, Hakeberg M, Kvist T, et al. Treatment of pulp in teeth affected by deep caries- a systematic review of the literature. Singapore Dental Journal. 2013;34:1-12.

6. Estrela C, Holland R, Estrela CR de A, Alencar HG, Sousa-Neto MD, et al. Characterization of successful root canal treatment. Brazilian Dental Journal. 2014;25(1):3-11.

7. Guo X, Miao H, Li L, Zhang S, Zhou D, Lu D, et al. Efficacy of four different irrigation techniques combined with $60^{\circ} \mathrm{C} 3 \%$ sodium hypochlorite and 17\% EDTA in smear layer removal. BMC Oral Health 2014;14:114-120.

8. Siqueira JF Jr. Aetiology of root canal treatment failure: why welltreated teeth can fail. Int Endod J. 2001;34:1-10.

9. Violich DR, Chandler NP. The smear layer in endodontics- a review. Internat Endodontic J. 2010;43:2-15.

10. Chu CH, Lo ECM, Cheung GSP. Outcome of root canal treatment using Thermafil and cold lateral condensation filling techniques. Int Endo J. 2005;38:179-185.
11. Saunder WP, Saunder EM. Coronal leakage as a cause of failure in rootcanal therapy: a review. Endod Dent Traumatol. 1994;10:105-108.

12. Carrotte P. Endodontic treatment for children.British Dental Journal 2005;198(1):9-15.

13. Colombo JS, Moore AN, Hartgerink JD, D’Souza RN. Scaffolds to control inflammation and facilitate dental pulp regeneration. J Enddod. 2014;40:S6-S12.

14. Shababang S. Treatment options: apexogenesis and apexification. J Endod. 2013;39:526-529.

15. Li Y, Shu LH, Yan M, Dai WY, Li JJ, Zhang GD, et al. Adult stem cell-based apexogenesis. World J Methodol. 2014;4(2):99-108.

16. Friedenstein AJ, Chailakhjan RK, Lalykina KS. The development of fibroblast colonies in monolayer cultures of guinea-pig bone marrow and spleen cells. Cell Tissue Kinet. 1970;3:393-403.

17. Caplan AI. Mesenchymal stem cells. J Orthop Res. 1991;9:641-650.

18. Ravindran S, Huang C-C and George A. Extracellular matrix of dental pulp stem cells : applications in pulp tissue engineering using somatic MSCs. Front Physiol . 2014; 4,article 395 (1-11).

19. Ravindran S, George A. Biomimetic extracellular matrix mediated somatic stem cell differentiation : applications in dental pulp tissue regeneration. Front Physiol. 2015,6:118;(1-9).

20. Hirata A, Dimitrova-Nakov S, Djole S-X, Ardila H, Baudry A, Kellermann $O$, et al. Plithotaxis, a collective cell migration, regulates the sliding of proliferating pulp cells located in the apical niche. Connective Tissue Research 2014;55(Suppl 1): 68-72.

21.Six N, Tompkins K, Septier D, Veis A, Goldberg M.Recruitment and characterization of the cells involved in reparative dentin formation in the exposed rat molar pulp after implantation of amelogenin gene splice products A+4 and A-4. Oral Biosciences \& Medicine. 2004;1:3544.

22. Simon S, Rilliard F, Berdal A, Machtou P. The use of mineral trioxide aggregate in one-visitt apexification treatment: a prospective study. Int Endo J. 2007;40:186-197.

23. Saito K, Nakatomi M, Ida-Yonemochi H, Ohshima H. Osteopontin is essential for type I collagen secretion in reparative dentin. J Dent Res. 2016:1-8.

24. Ravindran S, Zhang Y, Huang C-C, George A. Odontogenic induction of dental stem cells by extracellular matrix-inspired three-dimensional scaffold. Tissue Eng Part A. 2014;20(1-2):92-102.

25. Namour M, Theys S. Pulp revascularization of immature permanent teeth: a review of the literature and a proposal of a new clinical protocol. Scientific World Journal. 2014; Article ID 737503, 9 pages. 